\title{
ESTRESSORES EM UMA UNIDADE PÓS-OPERATÓRIA DE CIRURGIA TORÁCICA: AVALIAÇÃO DA ENFERMAGEM
}

\author{
Cristiane Franca Lisboa Gois ${ }^{1}$ \\ Rosana Aparecida Spadoti Dantas ${ }^{2}$
}

Gois CFL, Dantas RAS. Estressores em uma unidade pós-operatória de cirurgia torácica: avaliação da enfermagem. Rev Latino-am Enfermagem 2004 janeiro-fevereiro; 12(1):22-7.

Esse estudo descritivo foi realizado com o objetivo de identificar quais são os fatores geradores de estresse para pacientes internados em unidades pós-operatórias de cirurgias torácicas, segundo avaliação dos profissionais de enfermagem. Os dados foram coletados em dois hospitais, através de questionários preenchidos por membros das equipes de enfermagem que atuavam nas unidades pós-operatórias de cirurgias torácicas. Foi utilizada escala tipo Likert de 4 pontos para avaliar 42 possíveis estressores. A amostra constou de 58 profissionais, com tempo médio de atuação nas unidades de 5,9 anos. A média dos itens foi de 2,97, caracterizando-os entre pouco estressantes e estressantes. Os fatores mais estressantes foram: ter dor, ter tubos no nariz e/ou boca, estar amarrados por tubos e não conseguir dormir. Concluímos que os principais estressores para os pacientes, segundo avaliação dos profissionais da enfermagem, são aqueles relacionados ao procedimento cirúrgico.

DESCRITORES: estresse; unidades de terapia intensiva; cirurgia torácica; enfermagem

\section{STRESSORS IN CARE AT A THORACIC SURGERY POSTOPERATIVE UNIT: NURSING EVALUATION}

The aim of this descriptive study was to determine what events are perceived as stressful to patients in the thoracic surgery postoperative unit, according to nursing evaluation. Data were collected at two hospitals, where the intensive care nursing team members completed the questionnaire. A 4-point Likert scale was used to evaluate 42 possible stressors. The sample consisted of 58 nursing professionals. The average duration of their professional activities at the intensive care unit was 5.9 years. The average stressfulness score of the items was 2.97 (from little stressful to stressful). The most important stressors were: having pain, having tubes in nose and/or mouth, being tied down by tubes and not being able to sleep. We concluded that, according to nursing evaluation, the main stressors for patients are associated with the surgical procedure.

DESCRIPTORS: stress; intensive care units; thoracic surgery; nursing

\section{CAUSAS DE ESTRÉS EN UNA UNIDAD POST-OPERATORIA DE CIRUGÍAS TORÁXICAS: EVALUACIÓN DE ENFERMERÍA}

Este estudio descriptivo fue realizado con el objetivo de identificar cuales son los factores que generan estrés en pacientes internados en unidades post-operatorias de cirugías toráxicas, según la evaluación de los profesionales de enfermería. Los datos fueron recolectados en dos hospitales, por medio de cuestionarios diligenciados por los miembros de equipos de enfermería que trabajaban en estas unidades. Se utilizó una escala tipo Likert de 4 puntos para evaluar 42 posibles causas de estrés. La muestra constó de 58 profesionales, con tiempo promedio de actuación en las unidades de 5,9 años. El promedio de los ítems fue de 2,97 caracterizándolos entre poco estresantes y estresantes. Los factores más estresantes fueron: presencia de dolor, tener tubos en la nariz y/o boca, estar amarrados por tubos y no conseguir dormir. Concluimos que las principales causas de estrés para los pacientes, según la evaluación de los profesionales de enfermería, son aquellos relacionados con el procedimiento quirúrgico.

DESCRIPTORES: estrés; unidades de terapia intensiva; cirugía torácica; enfermería

\footnotetext{
${ }_{1}^{1}$ Enfermeira do Hospital das Clínicas da Faculdade de Medicina de Ribeirão Preto, da Universidade de São Paulo, e-mail: cristianeflg@hotmail.com;

${ }^{2}$ Enfermeira, Professor Doutor da Escola de Enfermagem de Ribeirão Preto, da Universidade de São Paulo, Centro Colaborador da OMS para o desenvolvimento da pesquisa em enfermagem, e-mail: rsdantas@eerp.usp.br
} 
INTRODUÇ̃̃O

As unidades pós-operatórias de cirurgia cardíaca são caracterizadas como centro de terapia intensiva. $O$ objetivo dessas unidades especializadas é promover o cuidado aos pacientes depois do ato anestésico-cirúrgico. A complexidade do atendimento prestado, bem como a estrutura física, o barulho, os equipamentos e a movimentação das pessoas são tidos como fontes geradoras de estresse para os pacientes e familiares ${ }^{(1)}$.

Estresse é qualquer situação na qual uma demanda não específica exige que o indivíduo reaja e tome uma atitude. É uma situação tensa, fisiológica ou psicológica, que pode afetar a pessoa em todas as dimensões humanas. A resposta ao estresse é influenciada pela intensidade, duração e âmbito do estressor e pelo número de estressores presentes no momento. Estressores são definidos como sendo estímulos precedentes ou precipitantes de mudança. Podem ser classificados em internos (aqueles que se originam dentro da pessoa como, por exemplo, uma febre) ou externos (aqueles que se originam fora da pessoa como, por exemplo, mudanças ambientais ou nas relações sociais) ${ }^{(2)}$. Assim podemos considerar que, em uma unidade de recuperação pós-operatória de cirurgia cardíaca, encontramos estímulos externos que estão presentes em seu ambiente físico e social e que podem ser fontes de estresse para os doentes.

Os pacientes submetidos à cirurgia cardíaca, de acordo com nossa experiência, têm um tempo de permanência nas unidades especializadas que varia de 48 a 72 horas. Durante esse período é normal ocorrerem situações que podem desencadear largo espectro de perturbações comportamentais. Pacientes críticos, em virtude de sua permanência nas unidades de terapia intensiva podem desenvolver traumas psicológicos significativos $^{(3)}$.

Pesquisas apontam que os distúrbios psíquicos, observados em alguns pacientes no pós-operatório de cirurgia cardíaca, estão relacionados com a própria unidade de recuperação e as rotinas que aí são seguidas ${ }^{(4)}$. Estudo realizado com pacientes e familiares sobre o significado cultural, atribuído ao centro de terapia intensiva, constatou que esse local é considerado como desconhecido e assustador e que o momento da alta é tido como a liberdade dos pacientes para a vida ${ }^{(5)}$.

Durante a permanência do indivíduo nessa unidade são vários os fatores que podem Ihe causar desconforto, como já foi abordado anteriormente. Na tentativa de atenuar a tensão desse paciente, muitas vezes, o profissional da saúde age empiricamente, analisando o estresse do paciente através da sua avaliação pessoal. Alguns autores relatam $^{(6-8)}$ que a avaliação do paciente, quanto aos fatores estressantes em um centro de tratamento intensivo, não coincide com a avaliação dos profissionais que o assistem, sendo que os enfermeiros tendem a valorizar os estressores com maior intensidade do que os próprios pacientes. A explicação desse fato pode estar na projeção dos sentimentos da equipe no paciente. Os enfermeiros mostraram-se sensibilizados pelo sofrimento dos pacientes e exteriorizaram a constante pressão psicológica sob a qual trabalham. Os principais fatores estressantes para o paciente, na visão da equipe de enfermagem, foram: ter dor, ter tubos no nariz e/ou na boca, estar amarrado por tubos e não conseguir dormir; enquanto que os pacientes apontaram como fatores mais estressantes: ter dor, não conseguir dormir, ter tubos no nariz e/ou na boca e não ter controle sobre si mesmo ${ }^{(9)}$.

Nossa realidade difere dos estudos citados e realizados em outros países ${ }^{(7-8)}$, no que se refere à presença de outros profissionais, além do enfermeiro, para a prestação da assistência de enfermagem. Apenas um estudo incluiu, além dos enfermeiros, os auxiliares de enfermagem em sua amostra. No entanto, os autores apresentaram os resultados não fazendo a discriminação entre os profissionais de enfermagem quanto à percepção do estresse para o paciente ${ }^{(9)}$.

A equipe de enfermagem representa o elo mais forte entre o paciente e o ambiente da unidade em que se encontra, por serem esses profissionais aqueles que, por maior tempo, exercem atividades junto ao paciente. Por isso, a humanização das unidades de terapia intensiva está intimamente vinculada à atuação dos profissionais de saúde frente aos fatores estressantes ${ }^{(9)}$. O cuidado de enfermagem é o ponto chave da hospitalização, pois permite estabelecer relações que contribuem para aliviar as fontes geradoras de estresse para os pacientes e seus familiares ${ }^{(5)}$. 
Diante disso, optamos por realizar nosso estudo com todos os profissionais da equipe de enfermagem com o objetivo de identificar quais são os fatores geradores de estresse para os pacientes submetidos à cirurgia cardíaca e internados em uma unidade pós-operatória especializada, segundo a perspectiva dos profissionais. Em outro estudo, iremos avaliar os fatores estressantes nessas unidades pós-operatórias de cirurgia cardíaca, segundo a opinião dos pacientes atendidos por esses profissionais.

\section{METODOLOGIA}

Delineamento do estudo: estudo descritivo e exploratório, realizado com profissionais de enfermagem em duas unidades pós-operatórias de cirurgias torácicas, entre os meses de junho e julho de 2002.

Local e população do estudo: a unidade pósoperatória, que descriminaremos pela letra $A$, pertence a um hospital público e de ensino e a unidade $B$ pertence a uma instituição hospitalar privada; ambas estão localizadas no município de Ribeirão Preto, Estado de São Paulo. Na ocasião da pesquisa, a unidade A constava de sete leitos e realizava, em média, 52 cirurgias por mês. A equipe de enfermagem que atuava no local era composta por: 16 enfermeiros, 01 técnico e 14 auxiliares de enfermagem. A unidade B destinava, em média, 06 leitos aos pacientes pós-operados de cirurgia cardíaca e realizava, aproximadamente, 40 cirurgias por mês e tinha 21 enfermeiros, 11 técnicos e 23 auxiliares de enfermagem. Em ambas as instituições, além dos enfermeiros lotados nas unidades pós-operatórias de cirurgias torácicas, outros enfermeiros do bloco cirúrgico e do hospital como um todo, assistiam, respectivamente, aos pacientes nas unidades A e $B$.

A amostra foi, por conveniência, sendo constituída por 58 profissionais de enfermagem que atuavam nessas duas unidades. Na unidade A, 13 dos 16 enfermeiros fizeram parte da amostra. Na unidade $B$, dos 21 enfermeiros, apenas 8 fizeram parte da amostra em virtude de atuarem mais constantemente prestando assistência ao paciente no pós-operatório de cirurgia cardíaca. Por esse mesmo motivo, dos 11 técnicos e 23 auxiliares de enfermagem, foram incluídos, respectivamente, 8 e 15 profissionais. Nossa amostragem foi limitada por alguns profissionais estarem de férias no período de coleta dos dados e pela não aceitação em participar do estudo, respondendo ao questionário.

Proteção dos sujeitos envolvidos no estudo: o projeto foi, primeiramente, aprovado pelo Comitê de Ética em Pesquisa do hospital onde estava localizada a unidade A e, posteriormente, pelo diretor técnico da segunda instituição hospitalar (unidade B). O objetivo do estudo foi apresentado para os profissionais de enfermagem e, com a concordância dos mesmos, o Termo de Consentimento Livre e Esclarecido foi assinado.

Coleta dos dados: os dados foram coletados através de questionários preenchidos anonimamente por cada um dos participantes. Os questionários continham duas partes, uma com dados sociodemográficos (idade, sexo, categoria profissional, tempo de atuação na unidade) e a outra contendo a "Escala de Estressores em Terapia Intensiva", em sua versão traduzida para o português ${ }^{(6,9)}$. O referido instrumento foi desenvolvido com 42 questões que abordam diversos fatores geradores de estresse em uma unidade de terapia intensiva ${ }^{(7,10)}$. Trata-se de escala do tipo Likert de quatro pontos, no qual (1) significa não estressante, (2) pouco estressante, (3) estressante, (4) muito estressante.

Análise dos dados: para a análise dos dados foi utilizado o programa Statistical Package for Social Science (SPSS) versão 10,0. Os dados sofreram análises descritivas e a consistência interna da escala usada foi verificada pelo alfa de Cronbach. A média dos escores foi calculada para cada um dos 42 estressores, classificados desde o mais estressante até o menos estressante. Para comparar os resultados obtidos pela escala de estressores entre enfermeiros e os demais profissionais de enfermagem (técnicos e auxiliares), usamos o teste nãoparamétrico de Mann-Whitney. O nível de significância estabelecido foi de 0,05 .

\section{RESULTADOS E DISCUSSÃO}

Com relação ao instrumento escolhido para avaliar os estressores nos pacientes internados em unidades de 
pós-operatório de cirurgias cardiotorácicas, obtivemos que a "Escala de Estressores em Terapia Intensiva"(9) mostrouse confiável para medir um conjunto de 42 fatores geradores de estresse. $\mathrm{O}$ alfa de Cronbach, obtido para o instrumento, foi de 0,90 e reflete a alta consistência interna dos itens da escala. Os estudos que mostraram a validação da escala para a língua portuguesa não apresentaram qual foi a confiabilidade da escala na população estudada ${ }^{(6,9)}$.

Foram entrevistados 58 profissionais de enfermagem, sendo que 27 (46,6\%) eram profissionais que atuavam na unidade A e 31 (53,4\%) na unidade B. No que se refere às categorias profissionais tivemos: 20 (34,5\%) enfermeiros, 8 (13,8\%) técnicos e 29 (50\%) auxiliares de enfermagem, um participante não completou esse item.

Visando a melhor caracterização dos profissionais enfermeiros e não enfermeiros quanto aos aspectos sociodemográficos e da percepção dos estressores (obtida pela Escala de Estressores em Terapia Intensiva) nas unidades onde atuavam, apresentaremos a estatística descritiva dessas variáveis na Tabela 1.

Tabela 1 - Estatística descritiva das variáveis do estudo, segundo as categorias enfermeiros e técnicos/auxiliares de enfermagem. Ribeirão Preto, 2003

\begin{tabular}{|c|c|c|c|c|}
\hline \multirow{2}{*}{$\begin{array}{c}\text { Categoria } \\
\text { profissional }^{*}\end{array}$} & \multicolumn{2}{|c|}{ Enfermeiros $(\mathrm{n}=20)$} & \multicolumn{2}{|c|}{ Técnicos e auxiliares $(n=37)$} \\
\hline & $\begin{array}{l}\text { Intervalo } \\
\text { (média) }\end{array}$ & $\mathrm{n}(\%)$ & $\begin{array}{l}\text { Intervalo } \\
\text { (média) }\end{array}$ & $\mathrm{n}(\%)$ \\
\hline $\begin{array}{l}\text { Escala estressores (soma } \\
\text { dos } 42 \text { itens) }\end{array}$ & $114-152(130)$ & & $85-153(121)$ & \\
\hline Idade (anos) & $23-51(35)$ & & $22-49(34,3)$ & \\
\hline $\begin{array}{l}\text { Tempo de atuação na } \\
\text { unidade (anos) }\end{array}$ & $0,58-13(5,2)$ & & $0,17-17(6,3)$ & \\
\hline \multicolumn{5}{|l|}{ Sexo } \\
\hline Feminino & & 17 (85) & & $23(62,2)$ \\
\hline Masculino & & 03 (15) & & $14(37,8)$ \\
\hline \multicolumn{5}{|l|}{ Hospital onde atua } \\
\hline A & & $12(60)$ & & $15(40,5)$ \\
\hline B & & $08(40)$ & & $22(59,5)$ \\
\hline
\end{tabular}

* Variável considerada com n=57 (1 dado não respondido)

O intervalo possível para a escala era de $42(42$ questões $\times 1$ ) até 168 (42 × 4), sendo que, quanto maior o valor da somatória dos itens, maiores são os estressores possíveis nas unidades de tratamento intensivo. $\mathrm{Na}$ amostra estudada obtivemos valores entre 114 e $152(\bar{x}=$ $129,6)$ para os enfermeiros e entre 85 e $153(\bar{x}=121)$ para os técnicos e auxiliares de enfermagem. Assim, constatamos que os enfermeiros avaliaram os itens como mais estressantes, quando comparados com os outros profissionais. Para testar se essa diferença era estatisticamente significante utilizamos o teste nãoparamétrico de Mann-Whitney, o qual constatou haver diferença estatística entre esses profissionais $(p<0,05)$. Todavia, não foi possível compararmos nosso resultado com aquele obtido em outro estudo ${ }^{(9)}$ realizado no Brasil, com profissionais de enfermagem, pois embora os autores tenham feito referência à análise, através da somatória dos itens da escala, eles não discriminaram os valores obtidos entre os diferentes profissionais entrevistados ${ }^{(9)}$.

Podemos observar que os dois grupos, enfermeiros e técnicos/auxiliares, não apresentaram diferenças importantes quanto à idade dos profissionais e o tempo de atuação nas duas instituições hospitalares envolvidas na pesquisa. O predomínio de profissionais do sexo feminino foi constatado nos dois grupos. Para comparar as pontuações dadas aos itens da escala pelos profissionais que atuam nas duas unidades, apresentaremos a seguir a Tabela 2 com os escores dos fatores geradores de estresse. 
Tabela 2 - Posição dos estressores em unidades pós-operatórias de cirurgias cardíacas, segundo os profissionais da enfermagem. Ribeirão Preto, 2003

\begin{tabular}{|c|c|c|c|c|c|c|}
\hline \multirow[t]{3}{*}{ Estressor } & \multicolumn{3}{|c|}{ Enfermeiros } & \multicolumn{3}{|c|}{ Técnicos/Auxiliares } \\
\hline & Posição & média & DP & Posição & & \\
\hline & & $(x)$ & (s) & & $(x)$ & (s) \\
\hline Estar amarrado por tubos & 01 & 3,80 & 0,69 & 03 & 3,60 & 0,65 \\
\hline Ter tubos no nariz e/ou na boca & 02 & 3,80 & 0,52 & 02 & 3,70 & 0,66 \\
\hline Não ter explicações sobre o tratamento & 03 & 3,75 & 0,55 & 08 & 3,40 & 0,64 \\
\hline Ter dor & 03 & 3,75 & 0,55 & 01 & 3,83 & 0,37 \\
\hline Não conseguir dormir & 04 & 3,70 & 0,47 & 05 & 3,54 & 0,80 \\
\hline Ter sede & 05 & 3,60 & 0,69 & 09 & 3,35 & 0,85 \\
\hline Escutar o barulho e os alarmes dos equipamentos & 06 & 3,50 & 0,51 & 12 & 3,21 & 0,71 \\
\hline Ter a equipe falando termos incompreensíveis & 07 & 3,45 & 0,68 & 22 & 2,86 & 0,94 \\
\hline Não ter privacidade & 08 & 3,42 & 0,54 & 13 & 3,18 & 0,84 \\
\hline Não ter controle de si mesmo & 09 & 3,40 & 0,68 & 06 & 3,45 & 0,64 \\
\hline Ter máquinas estranhas ao redor & 10 & 3,40 & 0,68 & 23 & 2,83 & 0,95 \\
\hline $\begin{array}{l}\text { Não conseguir mexer as mãos ou os braços devido às } \\
\text { vias intravenosas }\end{array}$ & 11 & 3,40 & 0,59 & 10 & 3,32 & 0,70 \\
\hline Escutar os alarmes do monitor cardíaco despertarem & 12 & 3,40 & 0,50 & 14 & 3,16 & 0,83 \\
\hline Ser furado por agulhas & 12 & 3,40 & 0,50 & 04 & 3,59 & 0,59 \\
\hline Sentir falta do marido ou da esposa & 13 & 3,35 & 0,67 & 13 & 3,18 & 0,84 \\
\hline Não saber onde está & 14 & 3,30 & 0,73 & 07 & 3,45 & 0,69 \\
\hline Enfermagem e médicos falando muito alto & 15 & 3,25 & 0,63 & 11 & 3,21 & 0,82 \\
\hline Escutar o gemido de outros pacientes & 15 & 3,25 & 0,63 & 15 & 3,16 & 0,79 \\
\hline $\begin{array}{l}\text { Sentir que a enfermagem está mais atenta aos } \\
\text { equipamentos do que a você }\end{array}$ & 16 & 3,20 & 0,95 & 24 & 2,78 & 0,82 \\
\hline $\begin{array}{l}\text { Assistir aos cuidados médicos e de enfermagem } \\
\text { realizados em outros pacientes }\end{array}$ & 17 & 3,20 & 0,61 & 19 & 2,97 & 0,95 \\
\hline Ter luzes acesas constantemente & 17 & 3,20 & 0,61 & 21 & 2,89 & 0,95 \\
\hline Cama e/ou travesseiros inconfortáveis & 18 & 3,15 & 0,58 & 18 & 3,00 & 0,74 \\
\hline Sons e ruídos desconhecidos & 19 & 3,10 & 0,55 & 27 & 2,62 & 0,79 \\
\hline Estar num ambiente muito quente ou muito frio & 20 & 3,05 & 0,82 & 17 & 3,00 & 0,78 \\
\hline Não saber quando as coisas vão ser feitas & 21 & 3,05 & 0,51 & 19 & 2,97 & 0,95 \\
\hline Sentir que a enfermeira está muito apressada & 21 & 3,05 & 0,51 & 20 & 2,91 & 0,89 \\
\hline Ver a família e os amigos por apenas alguns minutos & 22 & 3,00 & 0,72 & 28 & 2,59 & 0,83 \\
\hline Ser cuidado por médicos desconhecidos & 23 & 2,95 & 0,82 & 16 & 3,02 & 1,01 \\
\hline Ter que ficar olhando para os detalhes do teto & 23 & 2,95 & 0,82 & 20 & 2,91 & 0,89 \\
\hline Escutar o telefone tocar & 24 & 2,90 & 0,71 & 37 & 2,05 & 0,81 \\
\hline $\begin{array}{l}\text { Ser examinado por médicos e enfermeiros } \\
\text { constantemente }\end{array}$ & 25 & 2,75 & 0,91 & 30 & 2,40 & 1,03 \\
\hline Não saber que horas são & 26 & 2,75 & 0,85 & 34 & 2,21 & 0,94 \\
\hline Ser acordado pela enfermagem & 27 & 2,65 & 0,81 & 33 & 2,24 & 0,92 \\
\hline Não saber que dia é hoje & 28 & 2,65 & 0,74 & 26 & 2,70 & 0,93 \\
\hline Ser incomodado & 29 & 2,60 & 0,68 & 25 & 2,72 & 0,65 \\
\hline Sentir cheiros estranhos & 29 & 2,60 & 0,68 & 31 & 2,32 & 0,78 \\
\hline A enfermeira não se apresentar pelo nome & 30 & 2,50 & 0,60 & 35 & 2,16 & 0,79 \\
\hline $\begin{array}{l}\text { Ter homens e mulheres internados no mesmo } \\
\text { ambiente }\end{array}$ & 31 & 2,45 & 0,75 & 39 & 1,91 & 0,82 \\
\hline Medir a pressão arterial muitas vezes ao dia & 32 & 2,45 & 0,68 & 36 & 2,13 & 0,75 \\
\hline Ver as bolsas de soros penduradas sobre a cabeça & 33 & 2,40 & 0,68 & 32 & 2,29 & 0,74 \\
\hline Ter que usar oxigênio & 33 & 2,40 & 0,68 & 29 & 2,54 & 0,86 \\
\hline $\begin{array}{l}\text { Ter enfermagem constantemente fazendo tarefas ao } \\
\text { redor do leito }\end{array}$ & 34 & 2,35 & 0,81 & 38 & 2,02 & 0,72 \\
\hline
\end{tabular}

Lembrando que os valores de estresse na escala variam de 1 (não estressante) até 4 (muito estressante), os fatores mais estressantes, para os enfermeiros, foram, em ordem decrescente de estresse: estar amarrado por tubos ( $\bar{x}=3,80)$, ter tubos no nariz e/ou na boca ( $\bar{x}=$ 3,80 ), ter dor $(\bar{x}=3,75)$ e não ter explicações sobre o tratamento $(\bar{x}=3,75)$. Enquanto para os técnicos e auxiliares, os aspectos mais estressantes foram: ter dor $(\bar{x}=3,83)$, ter tubos no nariz e/ou na boca $(\bar{x}=3,70)$ e estar amarrado por tubos $(\bar{x}=3,60)$.

Ao analisarmos os resultados acima podemos constatar que os aspectos salientados foram os mesmos para os diferentes profissionais. A presença de tubos no nariz e/ou boca e estar "amarrado por tubos" foram aspectos considerados entre estressante e muito estressante. Essa percepção dos profissionais de enfermagem vai ao encontro da avaliação de pacientes cardíacos cirúrgicos, os quais também consideraram esse 
item como uma das principais fontes de estresse na unidade de terapia intensiva ${ }^{(6-7,9,11)}$. Para pacientes estudados em uma instituição hospitalar privada esse item foi colocado como o quinto gerador de estresse na escala de 42 itens $^{(9)}$

Ter dor foi apontado como um dos fatores mais estressante, resultado também encontrado por outros pesquisadores, tanto na visão dos pacientes e dos familiares, quanto na avaliação da equipe de enfermagem ${ }^{(3,9-10)}$. A dor, sintoma intrínseco ao procedimento anestésico-cirúrgico, também é decorrente do estado psicológico e do ambiente da unidade de terapia intensiva ${ }^{(12)}$. Quando não tratada, a dor predispõe o paciente à instabilidade cardiovascular, maior consumo energético e protéico, redução do volume ventilatório, além de interromper o sono. A interrupção do sono constituiu fator estressante para o paciente na opinião dos profissionais, sendo o quarto item na escolha dos enfermeiros e o quinto na escolha dos técnicos/auxiliares. Sob nosso ponto de vista, não conseguir dormir, resultaria em maior desgaste físico dos pacientes, fadiga e diminuição da motivação para interagirem com o tratamento.

Embora os itens considerados mais estressantes para os profissionais de enfermagem sejam aqueles relacionados ao tratamento cirúrgico (tubos, dor), esses profissionais podem atuar sobre outros aspectos

\section{REFERÊNCIAS BIBLIOGRÁFICAS}

1. Moura MLD. Factores que inciden em la atención de los pacientes y experiencia de apoyo psicológico como posible solución. In: Chaparro E, Infante E. Terapia Intensive y Unidad Coronária. Buenos Aires: Editorial Medica Panamericana; 1973. p.106-12.

2. Potter PA, Perry AG. Fundamentos de Enfermagem. Conceitos, processos e práticas. 4 ed. Rio de Janeiro (RJ): Guanabara Koogan; 1999.

3. Seidler, HE, Moritz R. Recordações dos principais fatores que causam desconforto nos pacientes durante a sua internação em uma Unidade de Terapia Intensiva. Rev Bras Terapia Intensiva 1998; 10(3):112-8.

4. Kornfeld DS, Heller SS, Frank KA, Wilson SN, Malm JR. Psychological and behavioral responses after coronary artery bypass surgery. Circulation 1982; 66 (Suppl 3):24.

5. Lemos RCA, Rossi LA. O significado cultural atribuído ao centro de terapia intensiva por clientes e seus familiares: um elo entre a beira do abismo e a liberdade. Rev Latino-am Enfermagem 2002 maio-junho; 10(2):345-57.

6. Novaes MA, Aronovich A, Ferraz M, Knobel E. Stressors in ICU: patients' evaluation. Intensive Care Med 1997; 23:12825.

Recebido em: 27.2.2003

Aprovado em: 28.7.2003 levantados na escala utilizada, de maneira a assegurar um ambiente que favoreça a recuperação dos doentes (diminuição de ruídos e luzes, fornecimento de explicações ao paciente com linguagem mais acessível.... ${ }^{(10)}$.

\section{CONCLUSÃO}

Nossa pesquisa procurou identificar quais os fatores estressantes ao paciente numa unidade pósoperatória de cirurgia cardíaca a partir da percepção dos profissionais de enfermagem. Os resultados sugerem que os principais estressores, na visão desses profissionais, foram aqueles relacionados ao procedimento anestésicocirúrgico, como ter tubos no corpo e sentir dor.

Comparando as respostas dadas por enfermeiros e por técnicos/auxiliares de enfermagem, quanto ao grau de estresse do total de 42 itens da Escala de Estressores em Terapia Intensiva, obtivemos diferença estatisticamente significante entre eles, sendo que os enfermeiros classificaram os itens como sendo mais estressantes do que os outros profissionais da enfemagem.

Entendemos que outros estudos necessitam ser conduzidos com os pacientes e familiares atendidos nas unidades envolvidas na pesquisa aqui apresentada, complementando os resultados obtidos na visão dos profissionais de enfermagem.

7. Cochran J, Ganong LH. A comparison of nurses' and patients' perception of intensive care unit stressors. J Adv Nurs 1989; 14(12):1038-43.

8. Cornock MA. Stress and the intensive care patient: perceptions of patients and nurses. J Adv Nurs 1998; 27(3):518-27.

9. Novaes MA, Knobel E, Bork AM. Estressores em UTI: Percepção do paciente, família e equipe de saúde. Intensive Care Med 1999; 25:1421-6.

10. Ballard KS. Identification of environmental stressors for patients in a surgical intensive care unit. Issues Ment Heath Nurs 1981; 1(3):89-108.

11. Soehren P. Stressors perceived by cardiac surgical patients in the intensive care unit Am J Crit Care 1995; 4(1):71-6.

12. Ferguson JÁ. Pain following coronary artery bypass grafting: an exploration of contributing factors. Intensive Crit Care Nurs 1992 Sep; 8(3):153-62. 